\title{
Quantitative Analysis of a New Method for Real-Time Generation of SNR Estimates for Digital Phase Modulation Signals
}

\author{
Yair Linn, Member, IEEE
}

\begin{abstract}
A new method of estimating the signal-to-noise ratio for M-PSK receivers was presented previously, which derives that estimate from the locked-state value of a new family of carrier lock detectors. In this paper, a quantitative analysis is undertaken in which it is shown that this method requires significantly less hardware and/or software resources than estimation from the received signal's error rate, is irrespective of the data sequence, and necessitates far fewer symbols in order to arrive at an equally accurate estimate.
\end{abstract}

Index Terms-Bit-error rate (BER), BPSK, carrier, detector, error rate, estimation, lock, metric, M-PSK, phase modulation, PSK, QPSK, real time, receiver design, signal-to-noise ratio (SNR), symbol error rate (SER).

\section{INTRODUCTION}

$\mathbf{I}$ $\mathrm{N}$ ANY M-PSK receiver, one of the most important metrics that that can be generated is an estimate of the channel $E_{S} / N_{0}$ ratio. In many modern communications schemes an accurate $E_{S} / N_{0}$ estimate is needed not only as a monitoring aid, but, rather, it plays an important role in the receiver's operation. For example, some error correction decoders can make use of an $E_{S} / N_{0}$ estimate to increase their coding gain (e.g. turbo codes [10]). Another example is systems that employ diversity reception, for which signal-to-noise ratio (SNR) estimates are used to assign relative weights to the data obtained from the various receivers. Yet another example is adaptive schemes where the data and/or coding rates are altered according to the $E_{S} / N_{0}$.

The need for $E_{S} / N_{0}$ estimates is not fulfilled merely by facilitating their availability; the estimates must also be timely. In this respect, the method analyzed here is shown to produce accurate estimates using only a small number of symbols, thus facilitating the generation of a rapidly updating estimate. Further advantages of the proposed method is that it is nondata aided, operates at a rate of one sample per symbol, and has a simple implementation that is compactly realized within a field programmable gate array (FPGA) or application specified integrated circuit (ASIC).

\section{REVIEW OF RECEIVER STRUCtURE AND LOCK DETECTOR}

\section{A. Signal and Receiver Models}

We denote the baseband M-PSK data signal as $m(t)=$ $\sum_{n=-\infty}^{\infty} a_{n} p(t-n T)$, where $a_{n}=\exp \left(j \phi_{n}\right), \phi_{n}=2 \pi$. $m_{n} / M, m_{n} \in\{0,1, \ldots, M-1\}$, and $p(t)$ is the baseband data

Manuscript received May 15, 2003; revised July 11, 2003 and July 21, 2003; accepted September 2, 2003. The editor coordinating the review of this paper and approving it for publication is J. K. Cavers. This work was supported in part by the University of British Columbia, by a Jean MacDonald Graduate Fellowship scholarship, and by the National Sciences and Engineering Research Council of Canada (NSERC).

The author is with the University of British Columbia, Vancouver BC V6T1Z4, Canada (e-mail: ylinn@ece.ubc.ca).

Digital Object Identifier 10.1109/TWC.2004.837428 pulse. The modulated signal is $s_{m}(t)=\operatorname{Re}\left[m(t) e^{j\left(\omega_{i} \cdot t+\theta_{i}\right)}\right]$ and that signal is corrupted by an additive white Gaussian noise (AWGN) channel. An M-PSK coherent receiver has the structure shown in Fig. 1, where we have the following.

1) $n(t) \sim N\left(0, N_{0} W\right)$, where $W$ is the width of the IF filter before the I-Q demodulator (not shown).

2) $K$ represents the total physical gain associated with the circuit. $K$ is, in general, a slow function of time, controlled by the automatic gain control (AGC) to achieve a desired signal level at the inputs of the samplers.

3) When the carrier loop is locked, $\Delta \omega=0$ and $\theta_{o} \in\left\{\theta_{i}-\right.$ $\left.\theta_{e}+2 \pi k / M \mid k=0,1, \ldots, M-1\right\}$, where $\left|\theta_{e}\right|<\pi / M$ is the residual phase error and $2 \pi k / M$ is the inherent carrier synchronization ambiguity.

4) The matched filters $h(t)$ are considered ideal, the sampling of their outputs is assumed to occur at the ideal time (i.e., the symbol synchronization loop is assumed locked), and the Nyquist criterion for zero-ISI [2] is assumed to hold regarding the post-matched filter waveforms.

\section{B. Lock Detector Definition, Expectation, and Distribution}

First, we define a process

$$
\begin{aligned}
x_{M, n} & \triangleq \frac{\operatorname{Re}\left[(I(n)+j \cdot Q(n))^{M}\right]}{\left(I^{2}(n)+Q^{2}(n)\right)^{\frac{M}{2}}} \\
& =\frac{\sum_{k=0}^{\frac{M}{2}}\left(\begin{array}{l}
M \\
2 k
\end{array}\right)(-1)^{k} I^{M-2 k}(n) Q^{2 k}(n)}{\left(I^{2}(n)+Q^{2}(n)\right)^{\frac{M}{2}}} .
\end{aligned}
$$

A new type of lock detector was defined in [1] through

$$
\hat{l}_{M, N}=\frac{1}{2 N} \sum_{n=-N+1}^{N} x_{M, n} .
$$

When the carrier loop is unlocked, it was shown in [1] that $E\left[\hat{l}_{M, N}\right]=0$. Conversely, when locked [1]

$$
f_{M}\left(\frac{E_{S}}{N_{0}}\right) \triangleq E\left[\hat{l}_{M, N}\right]=E[\cos (M \Delta \phi)] E\left[\cos \left(M \theta_{e}\right)\right]
$$

where $|\Delta \phi| \leq \pi$ and has the Rician phase distribution ([2], [11])

$$
\begin{aligned}
p(\Delta \phi)= & \frac{1}{2 \pi} \exp \left(\frac{-E_{S}}{N_{0}}\right) \\
\times & {\left[1+\sqrt{\frac{2 E_{S}}{N_{0}}} \cos (\Delta \phi) e^{\frac{E_{S}}{N_{0}} \cdot \cos ^{2}(\Delta \phi)}\right.} \\
\cos (\Delta \phi) \sqrt{\frac{2 E_{S}}{N_{0}}} & \left.\int_{-\infty}^{-\frac{x^{2}}{2}} d x\right] .
\end{aligned}
$$




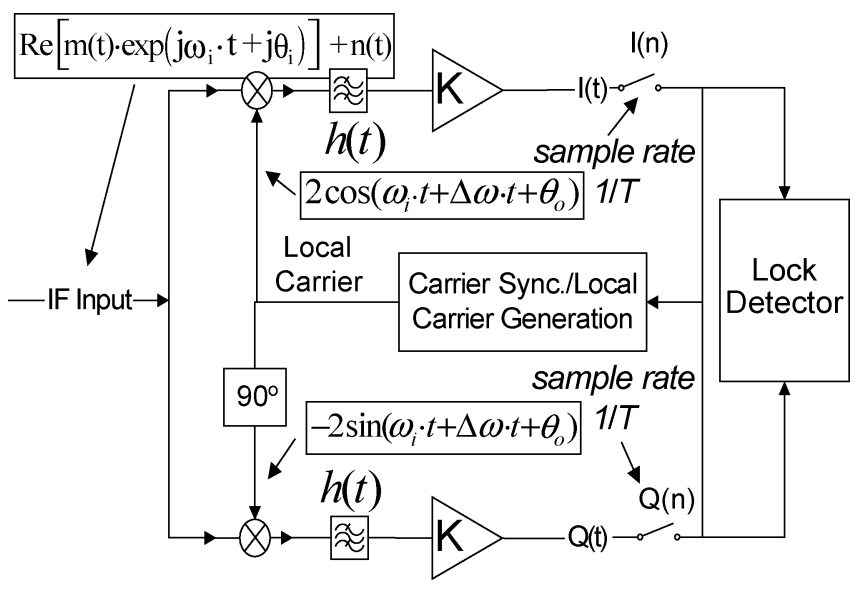

Fig. 1. Simplified M-PSK receiver schematic.

From the central limit theorem on (2), $\hat{l}_{M, N}$ has a Gaussian distribution, and, further

$$
\operatorname{Var}\left(\hat{l}_{M, N}\right)=\left(\frac{1}{2 N}\right) \cdot\left(E\left[x_{M, n}^{2}\right]-\left(E\left[x_{M, n}\right]\right)^{2}\right) .
$$

An important bound on the variance of $\hat{l}_{M, N}$ is also recalled from [1]

$$
\operatorname{Var}\left(\hat{l}_{M, N}\right) \leq \frac{1}{2 N} .
$$

Note that (6) is valid for both locked and unlocked states and for any phase error jitter conditions.

\section{Low Jitter Approximations}

Neglecting the effects of carrier phase jitter, (3) becomes

$$
f_{M}\left(\frac{E_{S}}{N_{0}}\right)=E[\cos (M \Delta \phi)]=\int_{-\pi}^{\pi} \cos (M \Delta \phi) p(\Delta \phi) d \Delta \phi .
$$

In this case, the variance of $\hat{l}_{M, N}$ is, from (5),

$$
\begin{aligned}
& \operatorname{Var}\left(\hat{l}_{M, N}\right)=\frac{1}{2 N} \\
& \quad \times\left(\int_{-\pi}^{\pi} \cos ^{2}(M \Delta \phi) p(\Delta \phi) d \Delta \phi-\left(f_{M}\left(\frac{E_{S}}{N_{0}}\right)\right)^{2}\right) .
\end{aligned}
$$

\section{III. $\mathrm{E}_{\mathrm{S}} / \mathrm{N}_{0}$ Estimation From $\hat{l}_{M, N}$}

\section{A. Theoretical Basis}

$f_{M}\left(E_{S} / N_{0}\right)$, given in (3) and (7), is a monotonically increasing function [1] and is thus invertible. It is this inverse relation $\gamma=f_{M}^{-1}\left(\hat{l}_{M, N}\right)$, namely the estimation of the $E_{S} / N_{0}$ ratio from the value of $\hat{l}_{M, N}$, which interests us in this paper. The $E_{S} / N_{0}$ estimate is usually desired in units of decibels, as follows:

$$
\gamma_{\mathrm{dB}}=10 \log _{10}\left(f_{M}^{-1}\left(\hat{l}_{M, N}\right)\right) .
$$

Fig. 2 shows simulated and predicted curves of (9). This figure was generated by first plotting $E\left[\hat{l}_{M, N}\right]=f_{M}\left(E_{S} / N_{0}\right)$ versus $\left(E_{S} / N_{0}\right)_{\mathrm{dB}}$, using the theoretical prediction of (7), as well as closed-loop simulations of $2 N=20000$ symbol

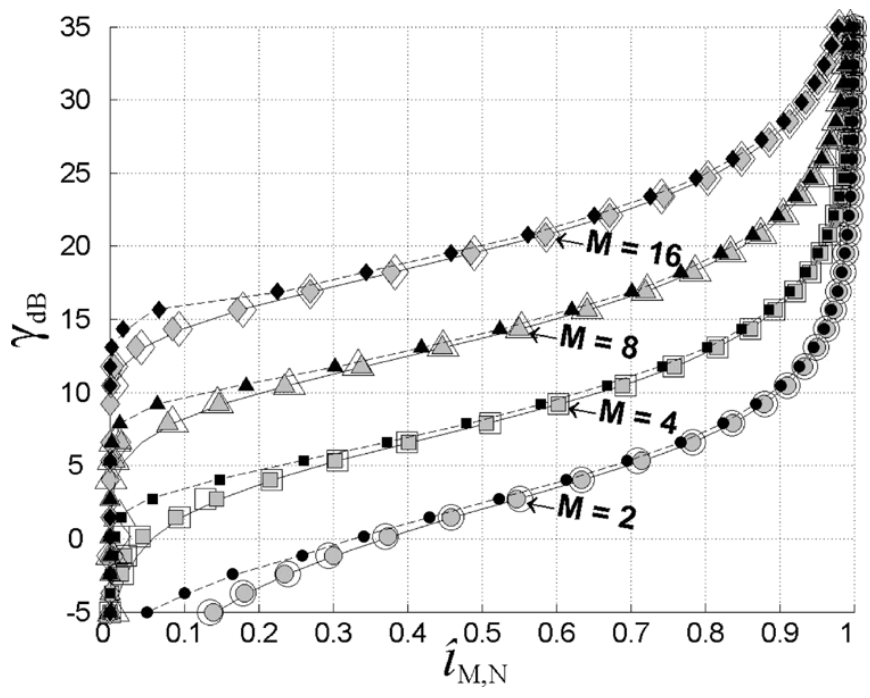

Fig. 2. Simulated and predicted values for (9).

intervals in which (2) was computed and (3) was approximated. Then, the graph was reflected through its $y=x$ diagonal to produce the inverse relation, as given in (9). Results are presented for second-order phase-locked loop (PLL) synchronizers with $2 B_{L} \cdot T=0.01$ and $2 B_{L} \cdot T=0.1$, where $B_{L}$ is the PLL's noise bandwidth $\left[B_{L}=0.5 \omega_{n}(\xi+1 /(4 \xi))\right.$, where $\omega_{n}$ is the natural radian frequency of the PLL, and $\xi$ is its damping factor (see [5])]. Specifically, in Fig. 2 the solid lines are plots for the theoretical jitter-free case of $f_{M}^{-1}(\bullet)$ [i.e., $f_{M}(\bullet)$ given by (7)]. The blank polygons in that figure were obtained in the closed-loop simulations using (2), where a normalized PLL noise bandwidth of $2 B_{L} \cdot T=0.01$ was employed. The gray polygons are values predicted by (3) with $2 B_{L} \cdot T=0.01$ and using the time average $\overline{\cos \left(M \theta_{e}\right)}$ to approximate $E\left[\cos \left(M \theta_{e}\right)\right]$, where $\theta_{e}$ was measured in the aforementioned simulations. Completing Fig. 2 are curves obtained with a normalized PLL noise bandwidth of $2 B_{L} \cdot T=0.1$, where the dashed lines were obtained in closed-loop simulations using (2), and the black polygons are values predicted by (3), with $\overline{\cos \left(M \theta_{e}\right)}$ approximating $E\left[\cos \left(M \theta_{e}\right)\right]$.

In carrier synchronization PLLs, the quantity $2 B_{L} \cdot T$ is rarely ([3], [5], [7]) larger than the order of magnitude of $2 B_{L} \cdot T=$ 0.01 and is virtually never as high as $2 B_{L} \cdot T=0.1$. Thus, it can be safely said (in lieu of Fig. 2) that it is permissible to always use (7) and not bother with trying to predict (3); this will henceforth be assumed. The case is less clear for using (8) when nonnegligible carrier phase error jitter is present; however, since (6) holds for any phase jitter conditions, it is easy to arrive at "worst case" bounds, which will also be given.

\section{B. Hardware Implementation}

Fig. 3 presents a structure for the generation of (9) in hardware. Since [1] $\left|x_{M, n}\right| \leq 1,\left|\hat{l}_{M, N}\right| \leq 1$, and since a small dynamic range is needed for (9) (see Fig. 2), the lookup tables can be realized as small fixed-point lookup tables, thus permitting efficient implementation of Fig. 3 within an FPGA or ASIC. Note how outright division by $2 N$ is avoided in Fig. 3, where for this to be accurate $N$ should be chosen to be a power of 


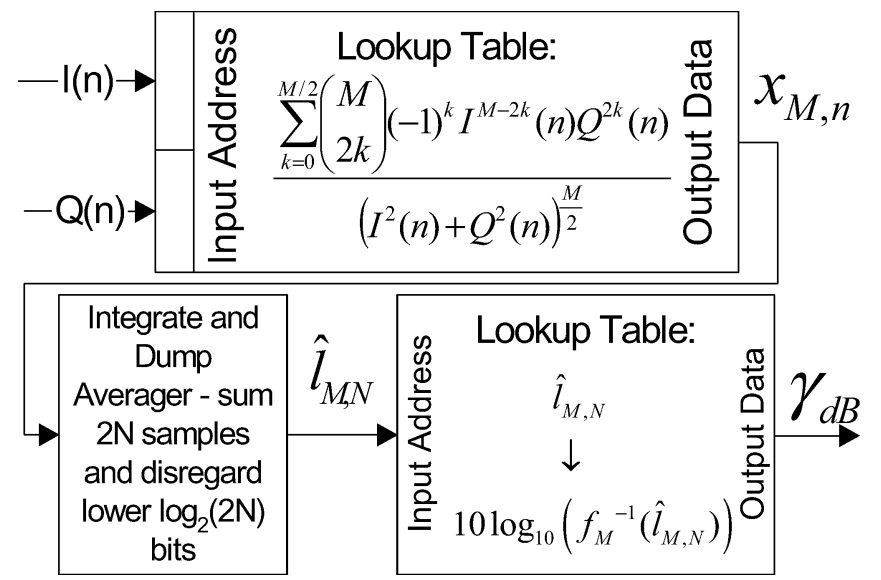

Fig. 3. Efficient hardware generation of $\gamma_{\mathrm{dB}}$.

2. Note also that if $\hat{l}_{M, N}$ is already generated for lock detection, then $E_{S} / N_{0}$ estimation requires merely the addition of the small lookup table implementing (9), a trivial addendum.

\section{Quantitative Investigation}

From (6) or (8) it is clear that in order to achieve a more accurate value of $\hat{l}_{M, N}$, and by extension of $\gamma=f_{M}^{-1}\left(\hat{l}_{M, N}\right), N$ should be increased until $\operatorname{Var}\left(\hat{l}_{M, N}\right)$ falls below an acceptable value. Indeed, the purpose of this paper may be stated as follows: we would like to compute the minimal value of $N$ needed to achieve a desired tolerance in the estimation of the $E_{S} / N_{0}$, with a desired confidence. Mathematically, the question is: What is the minimal value of $N$ needed so that the following holds?

$$
P\left(\left|f_{M}^{-1}\left(\hat{l}_{M, N}\right)-\frac{E_{S}}{N_{0}}\right|<\text { tol }\right)>C
$$

where in (10) tol is the tolerance and $C$ is the confidence. Assume tol is in units of decibels. We define the constants $r_{1}=$ $\left(10^{\text {tol } / 10}-1\right)$ and $r_{2}=\left(1-10^{- \text {tol } / 10}\right)$ (which describe the allowed deviations from the $\left.E_{S} / N_{0}\right)$. Since $f_{M}\left(E_{S} / N_{0}\right)$ is a monotonously increasing function, (10) implies

$$
\begin{aligned}
& P\left[-r_{2} \cdot \frac{E_{S}}{N_{0}}<f_{M}^{-1}\left(\hat{l}_{M, N}\right)-\frac{E_{S}}{N_{0}}<r_{1} \cdot \frac{E_{S}}{N_{0}}\right]>C \\
& \Leftrightarrow P\left[f_{M}\left[\left(1-r_{2}\right) \frac{E_{S}}{N_{0}}\right]<\hat{l}_{M, N}<f_{M}\left[\left(1+r_{1}\right) \frac{E_{S}}{N_{0}}\right]>C .\right.
\end{aligned}
$$

Now, defining

$$
\begin{aligned}
y \triangleq \min \left\{\left|f_{M}\left[\left(1-r_{2}\right) \frac{E_{S}}{N_{0}}\right]-f_{M}\left[\frac{E_{S}}{N_{0}}\right]\right|,\right. & \\
& \left.\left|f_{M}\left[\left(1+r_{1}\right) \frac{E_{S}}{N_{0}}\right]-f_{M}\left[\frac{E_{S}}{N_{0}}\right]\right|\right\}
\end{aligned}
$$

and using $E\left[\hat{l}_{M, N}\right]=f_{M}\left(E_{S} / N_{0}\right)$

$$
\begin{array}{r}
P\left[f_{M}\left[\left(1-r_{2}\right) \frac{E_{S}}{N_{0}}\right]<\hat{l}_{M, N}<f_{M}\left[\left(1+r_{1}\right) \frac{E_{S}}{N_{0}}\right]\right] \\
\geq P\left[\left|\frac{\hat{l}_{M, N}-E\left[\hat{l}_{M, N}\right]}{\sqrt{\operatorname{Var}\left(\hat{l}_{M, N}\right)}}\right|<\frac{y}{\sqrt{\operatorname{Var}\left(\hat{l}_{M, N}\right)}}\right] .
\end{array}
$$

Because $\hat{l}_{M, N}$ is Gaussian, it follows from (12) that in order for (10) to occur it is sufficient to require (see [2, ch. 2])

$$
\begin{aligned}
P\left(\left|\frac{\hat{l}_{M, N}-E\left[\hat{l}_{M, N}\right]}{\sqrt{\operatorname{Var}\left(\hat{l}_{M, N}\right)}}\right|\right. & \left.<\frac{y}{\sqrt{\operatorname{Var}\left(\hat{l}_{M, N}\right)}}\right) \\
& =\operatorname{erf}\left(\frac{y}{\sqrt{2} \sqrt{\operatorname{Var}\left(\hat{l}_{M, N}\right)}}\right)>C
\end{aligned}
$$

with $\operatorname{erf}(x)=(2 / \sqrt{\pi}) \int_{0}^{x} e^{-t^{2}} d t$. If carrier phase jitter is negligible, then using (8) in (13) and solving for $N$

$$
\begin{aligned}
2 N & >2\left(\frac{\operatorname{erf}^{-1}(C)}{y}\right)^{2} \\
& \times\left(\int_{-\pi}^{\pi} \cos ^{2}(M \Delta \phi) \cdot p(\Delta \phi) \cdot d \Delta \phi-\left(f_{M}\left(\frac{E_{S}}{N_{0}}\right)\right)^{2}\right) .
\end{aligned}
$$

Conversely, a worst case result accounting for phase error jitter may be obtained from (13) and (6)

$$
2 N>2\left(\frac{\operatorname{erf}^{-1}(C)}{y}\right)^{2} .
$$

(Note that for all the equations in this section $f_{M}\left(E_{S} / N_{0}\right)$ is given in (7). See comments made in the last paragraph of Section III-A.)

To recap, we have shown that choosing $N$ which complies with (14) or (15) ensures the verity of (10). Equations (14) and (15) are expressed in terms of $2 N$ (not simply of $N$ ) because $2 N$ is the number of symbols used in computation of the lock metric from which the $E_{S} / N_{0}$ is estimated [see (2)].

\section{DEVElopMEnT OF A COMPARISON YARDSTICK- $\mathrm{E}_{\mathrm{S}} / \mathrm{N}_{0}$ ESTIMATION VIA THE $S E R$}

A great proportion of modern communications systems produce SNR estimates by measuring the pre- or post-decoder error rate. For example, this is what is done in countless systems that estimate the SNR from the number of errors detected in preambles or training sequences that are embedded in the data stream. Thus, perhaps the most meaningful and universally applicable yardstick by which to measure the efficacy of SNR estimation via (9) is attained through comparison of (14) or (15) to the number of symbols needed for $E_{S} / N_{0}$ estimation via measurement of the pre-decoder symbol error rate (SER). This is because for coded signals, the post-decoder error rate is always smaller (often by orders of magnitude) than the pre-decoder SER, i.e., the number of symbols needed for SNR estimation via the pre-decoder SER can also be viewed as a lower bound for that which is needed for estimation via post-decoder error rates, regardless of the coding scheme used.

From [2], the uncoded M-PSK symbol error probability is

$$
P_{e}\left(M, \frac{E_{S}}{N_{0}}\right)=1-\int_{-\frac{\pi}{M}}^{\frac{\pi}{M}} p(\Delta \phi) \cdot d(\Delta \phi) \triangleq g_{M}\left(\frac{E_{S}}{N_{0}}\right)
$$

where (4) defines $p(\Delta \phi)$ (note that (16) ignores the effects of phase jitter on the SER. Equation (16) can still model such ef- 
fects by first incorporating them [4] into a reduced effective $E_{S} / N_{0}$, but Section V shows that the advantage of the method discussed in Section III is so great that any effects of such a minute correction are irrelevant). To avoid cumbersome notation, we denote the left-hand side of (16) simply as $P_{e}$. We define the binary auxiliary variables $U_{i}$ as $U_{i}=1 \Leftrightarrow$ an error was detected in symbol $i$. Assuming the errors in the received symbols occur randomly and independently, we have $P\left(U_{i}=1\right)=$ $P_{e}$ and $P\left(U_{i}=0\right)=1-P_{e}$, and therefore $E\left[U_{i}\right]=P_{e}$ and $\operatorname{Var}\left(U_{i}\right)=P_{e}\left(1-P_{e}\right)$. We can define the measured SER as $S(L)=(1 / L) \sum_{i=1}^{L} U_{i}$, and from the central limit theorem we then have

$$
S(L) \sim N\left(P_{e}, \frac{P_{e}\left(1-P_{e}\right)}{L}\right) .
$$

Since $E[S(L)]=P_{e}$ and thus $E\left[g_{M}^{-1}(S(L))\right]=E_{S} / N_{0}$, we can estimate the $E_{S} / N_{0}$ from $S(L)$ via $\eta=g_{M}^{-1}(S(L))$. For comparison to (14) and (15), we are interested in finding a value of $L$ that ensures that

$$
P\left[-r_{2} \cdot \frac{E_{S}}{N_{0}}<\eta-\frac{E_{S}}{N_{0}}<r_{1} \cdot \frac{E_{S}}{N_{0}}\right]>C
$$

where $r_{1}=\left(10^{\mathrm{tol} / 10}-1\right)$ and $r_{2}=\left(1-10^{-\mathrm{tol} / 10}\right)$. Developing (18) further, we have that it is equivalent to

$$
P\left[\left(1-r_{2}\right) \cdot \frac{E_{S}}{N_{0}}<g_{M}^{-1}(S(L))<\left(1+r_{1}\right) \cdot \frac{E_{S}}{N_{0}}\right]>C .
$$

Note that $g_{M}\left(E_{S} / N_{0}\right)$ is a monotonically decreasing function [2] (in other words, the probability of symbol error is inversely related to the $E_{S} / N_{0}$ ). Using this, we have that an equivalent requirement to (19) is

$$
P\left[g_{M}\left[\left(1-r_{2}\right) \cdot \frac{E_{S}}{N_{0}}\right]>S(L)>g_{M}\left[\left(1+r_{1}\right) \cdot \frac{E_{S}}{N_{0}}\right]\right]>C \text {. }
$$

Defining $z \triangleq \min \left\{\left|g_{M}\left(\left(1-r_{2}\right) \cdot E_{S} / N_{0}\right)-P_{e}\right|, \mid g_{M}\left(\left(1+r_{1}\right)\right.\right.$. $\left.\left.E_{S} / N_{0}\right)-P_{e} \mid\right\}$, we have that for (20) to be fulfilled it suffices that

$$
P\left(\left|\frac{S(L)-P_{e}}{\sqrt{\operatorname{Var}(S(L))}}\right|<\frac{z}{\sqrt{\operatorname{Var}(S(L))}}\right)>C .
$$

Equation (17) applied to (21) means that for (18) to be guaranteed it is sufficient to require

$$
L>\frac{2 P_{e}\left(1-P_{e}\right)}{\left(\frac{z}{\operatorname{erf}^{-1}(C)}\right)^{2}} .
$$

\section{Display AND ANALYSIS OF RESUlts}

A meaningful appraisal of the utility of estimating the $E_{S} / N_{0}$ via (9) may be obtained by comparing the number of symbols necessary for such an estimate, as per (14) or (15), to that which is required to attain a similarly accurate estimate from the SER,
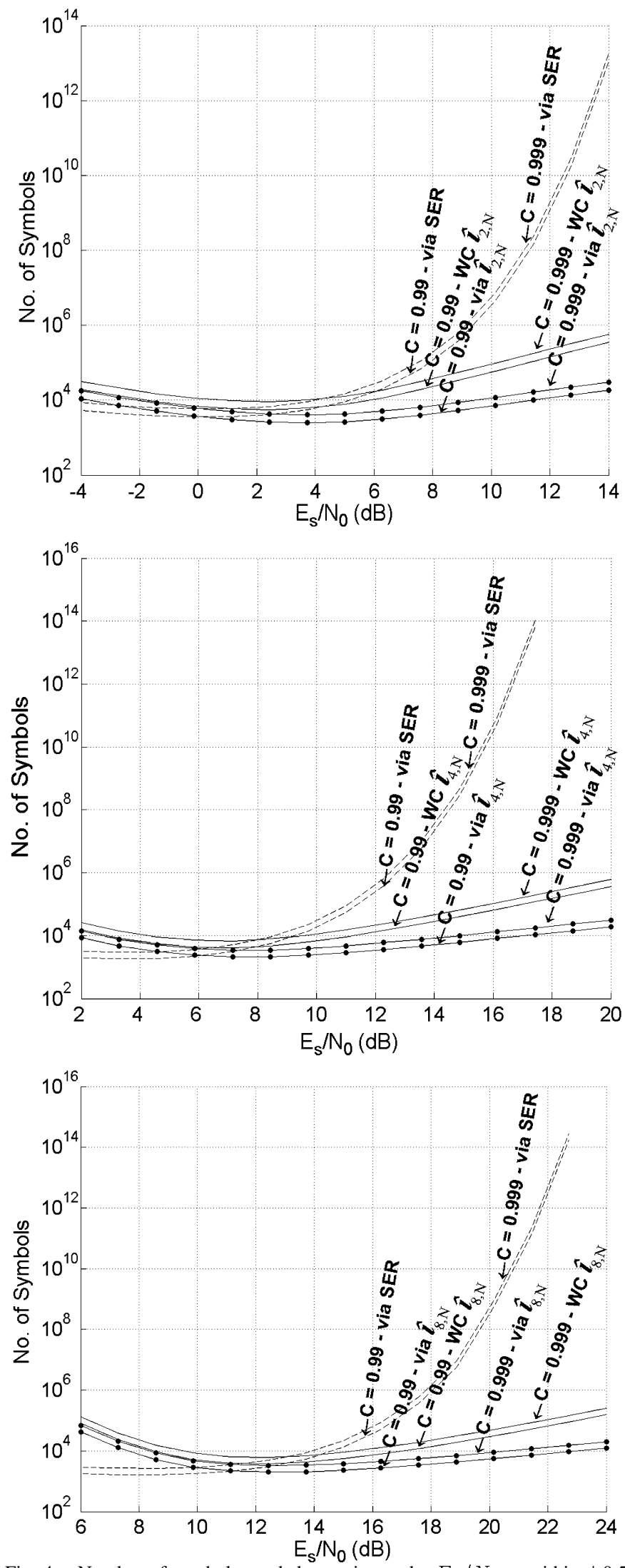

Fig. 4. Number of symbols needed to estimate the $E_{S} / N_{0}$ to within \pm 0.5 dB. Top: $M=2$ (BPSK); middle: $M=4$ (QPSK); bottom: $M=8$ (8-PSK). Curves labeled WC denote worst case results for $\hat{l}_{M, N}$, i.e. using (15). Other curves obtained using (14) and (22).

as per (22). This is shown in Fig. 4 for BPSK, QPSK, and 8-PSK, for $C=99 \%$ and $C=99.9 \%$, with tol $=0.5 \mathrm{~dB}$. As that 
figure clearly illustrates, estimation via $\hat{l}_{M, N}$ is particularly advantageous for higher $E_{S} / N_{0}$ ratios, where it is seen that while estimation via the SER experiences exponential growth in the number of symbols necessary, the growth rate for estimation from $\hat{l}_{M, N}$ is much milder.

Some comments are in order regarding Fig. 4 when the data stream sent is not a known sequence but is rather an arbitrary data stream. In that figure, it is seen that for low $E_{S} / N_{0}$ ratios, apparently estimation via the SER requires less symbols. However, this is a fallacy since Fig. 4 assumes that the SER is measured precisely. The only way to achieve a data-independent SER measurement is by first using an error correction decoder and then comparing the corrected data to the input data stream [8], thus arriving at the pre-decoder SER. This tacitly assumes that the post-decoder data stream is error free. Yet, at low $E_{S} / N_{0}$, the post-decoder data stream cannot be approximated as error free, thus inherently skewing the SER measurement. Indeed, the decoder may not even be in lock for low $E_{S} / N_{0}$ ratios, making the SER measurement impossible in the first place. "Training sequences," or known sequences of symbols, can be transmitted in order to arrive at an accurate SER estimate; however, this means that at least some of the channel throughput is taken up by such sequences. In contrast, estimation via $\hat{l}_{M, N}$ suffers no such impediments, as it is independent of the data stream, the decoding scheme, and the post-decoder error rate. Thus, Fig. 4 can be viewed as optimistic w.r.t. estimation via the SER, and consequently estimation via $\hat{l}_{M, N}$ can be considered superior for all $E_{S} / N_{0}$ ratios. Furthermore, estimation via the SER requires error detection and accrual mechanisms, which often necessitate nontrivial hardware and/or software resource appropriations. This is quite different from the situation discussed in Section III-B.

Several additional SNR estimation methods are presented in [8] and [9]. While a discussion of those methods is impossible within the span of the current paper, it shall be commented that of the methods in [8] and [9], some are unique to a specific receiver structure, most require symbol decisions to be made, and several require more than one sample per symbol. Furthermore, none of those methods appears to have a hardware implementation nearly as compact as the one suggested here.

As final points, it should be mentioned that, first, due to the resilience of $\hat{l}_{M, N}$ to effects stemming from imperfect AGC control of $K$ in Fig. 1 (see [1]), this resilience is also present in the resulting $E_{S} / N_{0}$ estimates, thus deeming them reliable even when rapidly fading signal conditions are encountered. Secondly, the proposed method is also applicable to the modulation schemes related to M-PSK that are discussed in [1], if the appropriate (sometimes modified) lock detector implementation is chosen (see [1]).

\section{CONCLUSION}

In this paper, an analysis of a new method of $E_{S} / N_{0}$ estimation for M-PSK receivers was undertaken, and quantitative formulas describing its performance were developed. It was found that the proposed method has several quantitative and qualitative advantages with respect to previously available methods. These include: independence from the received data, no reliance on symbol decisions or error detection, a simple and compact fixed-point hardware implementation, and, perhaps most importantly, it requires only a relatively small number of symbols to arrive at an accurate estimate. Thus, the method analyzed here is particularly well suited for real-time estimate generation.

\section{ACKNOWLEDGMENT}

The author would like to thank his supervisor, Prof. M. J. Yedlin, for his unyielding support and encouragement regarding the author's research endeavors. Furthermore, the author would like to express his gratitude to the editor Prof. J. Cavers and to the anonymous reviewers for their conscientious efforts.

\section{REFERENCES}

[1] Y. Linn and N. Peleg, "A family of self-normalizing carrier lock detectors and $E_{S} / N_{0}$ estimators for M-PSK and other phase modulation schemes," IEEE Trans. Wireless Commun., vol. 3, pp. 1659-1668, Sept. 2004.

[2] J. G. Proakis, Digital Communications, 4th ed. New York: McGrawHill, 2001.

[3] H. Meyr, M. Moeneclaey, and S. A. Fechtel, Digital Communication Receivers. New York: Wiley, 1997.

[4] M. K. Simon, S. M. Hinedi, and W. C. Lindsey, Digital Communications Techniques. Upper Saddle River, NJ: Prentice-Hall, 1994.

[5] F. M. Gardner, Phaselock Techniques, 2nd ed. New York: Wiley, 1979.

[6] W. P. Osborne and B. T. Kopp, "Synchronization in M-PSK modems," in Proc. ICC' 92, vol. 3, pp. 1436-1440.

[7] _ "An analysis of carrier phase jitter in an M-PSK receiver utilizing MAP estimation," in Proc. MILCOM '93, vol. 2, pp. 465-470.

[8] N. Celandroni, E. Ferro, and F. Potorti, "Quality estimation of PSK modulated signals," IEEE Commun. Mag., pp. 50-55, Jul. 1997.

[9] D. R. Pauluzzi and N. C. Beaulieu, "A comparison of SNR estimation techniques for the AWGN channel," IEEE Trans. Commun., vol. 48, pp. 1681-1691, Oct. 2000.

[10] T. A. Summers and S. G. Wilson, "SNR mismatch and online estimation in turbo decoding," IEEE Trans. Commun., vol. 46, pp. 421-423, Apr. 1998.

[11] H. Gudbjartsson and S. Patz, "The Rician distribution of noisy MRI data," Magnetic Resonance Medicine, vol. 34, no. 6, pp. 910-914, Dec. 1995.

[12] H. Gudbjartsson and S. Patz, "Erratum to the Rician distribution of noisy MRI data," Magnetic Resonance Medicine, vol. 36, pp. 331-333, Aug. 1996. 